\title{
Axial-vector nucleon-to-delta transition form factors using the complex-mass renormalization scheme
}

\author{
Y. Ünalø ${ }^{1}$ A. Küçükarslan, ${ }^{1}$ and S. Scherer ${ }^{2}$ \\ ${ }^{1}$ Physics Department, Çanakkale Onsekiz Mart University, 17100 Çanakkale, Turkey \\ ${ }^{2}$ Institut für Kernphysik, Johannes Gutenberg-Universität Mainz, D-55099 Mainz, Germany
}

(Received 8 April 2021; accepted 15 October 2021; published 10 November 2021)

\begin{abstract}
We investigate the axial-vector nucleon-to-delta transition form factors in the framework of relativistic baryon chiral perturbation theory at the one-loop order using the complex-mass renormalization scheme. We determine the available six free parameters by fitting to an empirical parametrization of the form factors obtained from the BNL neutrino bubble chamber experiments. A unique feature of our calculation is the prediction of a nonvanishing form factor $C_{3}^{A}\left(Q^{2}\right)$. Moreover, our results show a surprising sensitivity to the coupling constant $g_{1}$ of the leading-order Lagrangian $\mathcal{L}_{\pi \Delta}^{(1)}$.
\end{abstract}

DOI: 10.1103/PhysRevD.104.094014

\section{INTRODUCTION}

The $\Delta(1232)$ resonance is the first and best-established excitation of the nucleon [1]. In the static quark model, it consists of three constituent quarks, coupled to spin $S=\frac{3}{2}$ and isospin $I=\frac{3}{2}$. The dominant decay mode by far is the strong decay into a pion and a nucleon, resulting in a lifetime of the order of $10^{-23} \mathrm{~s}$. According to Ref. [1], the pole position in the complex-energy plane is at $z_{\Delta}=m_{\Delta}-i \frac{\Gamma_{\Delta}}{2} \approx$ $(1210-i 50) \mathrm{MeV}$.

While there is a substantial amount of empirical information on the electromagnetic (vector) nucleon-to-delta transition [2-15] (see, e.g., Refs. [16,17] for a review), very little is known about the axial-vector nucleon-to-delta transition [18-22]. The reason is twofold: (a) the weak probe couples only feebly to the nucleon-delta system and (b) the delta is unstable. ${ }^{1}$ On the theoretical side, numerous investigations exist for the electromagnetic case (see Ref. [23] and references therein) which have been extensively compared with data. Also, theoretical calculations of the axial-vector nucleon-to-delta transition have been performed in the framework of quark models [24-28],

\footnotetext{
${ }^{1}$ Of course, the second argument also applies to the electromagnetic transition. Therefore, our knowledge of the electromagnetic transition form factors is substantially less than for the nucleon elastic form factors. Concerning the transition from an unstable delta state to an unstable delta state, Ref. [1] quotes only a rough guess of the range, within which the magnetic moment is expected to lie.

Published by the American Physical Society under the terms of the Creative Commons Attribution 4.0 International license. Further distribution of this work must maintain attribution to the author(s) and the published article's title, journal citation, and DOI. Funded by SCOAP ${ }^{3}$.
}

chiral effective field theory [29-32], lattice QCD [33-35], and light-cone QCD sum rules [36,37].

While traditional calculations treat the delta resonance essentially as a stable particle, it was emphasized in Ref. [38] that form factors of unstable particles should be determined from the renormalized three-point function at the complex pole. In fact, this idea was applied in Ref. [23] to the electromagnetic nucleon-to- $\Delta$ resonance transition to third chiral order in manifestly Lorentzinvariant chiral effective field theory. At the pole position, the magnetic dipole, electric dipole, and Coulomb quadrupole form factors $G_{M}, G_{E}$, and $G_{C}$ are complex quantities. In particular, it was found that $G_{E}$ and $G_{C}$ have imaginary parts which are of the same magnitude as the respective real parts. In the present article, we extend the analysis to the axial-vector transition at the one-loop level. For that purpose, we combine a covariant description of the $\Delta(1232)$ resonance $[39,40]$ with the complex-mass scheme (CMS) applied to the chiral effective field theory of the strong interaction [41]. ${ }^{2}$

This article is organized as follows. In Sec. II, we introduce the axial-vector nucleon-to-delta transition process and discuss how it is related to weak pion production. In this context, we also define the pion-nucleon-delta form factor in terms of the PCAC relation (partially conserved axial-vector current). In Sec. III, we present the effective Lagrangians we used. In Sec. IV, we calculate the transition form factors and show our results. Section V contains a comparison with other work. In Sec. VI, we give a short summary.

\footnotetext{
${ }^{2}$ The CMS was originally developed for deriving properties of $W, Z$, and Higgs bosons obtained from resonant processes [42-46].
} 


\section{AXIAL-VECTOR NUCLEON-TO-DELTA TRANSITION FORM FACTORS}

\section{A. Weak pion production}

The $\Delta(1232)$ is an unstable particle with a very short lifetime of the order of $10^{-23} \mathrm{~s}$. Therefore, strictly speaking, stable one-particle states $|\Delta(p)\rangle$ with $p^{2}=m_{\Delta}^{2}$ do not exist [47]. For this reason, direct measurements of transition form factors are impossible, because the $\Delta(1232)$ is not an asymptotic state of the strong interactions. ${ }^{3}$ On the other hand, the existence of the delta is prominently seen in pion-nucleon scattering or pion photoproduction on the nucleon. In other words, the impact of an unstable $\Delta$ may be investigated in terms of a complete scattering amplitude, where it contributes as an intermediate "state." In the present case, we are interested in the axial-vector nucleon-to-delta transition. This may be studied in the weak production of a pion on the nucleon with hadronic center-of-mass energies in the delta region [48]. For kinematical conditions such that the square root of the Mandelstam variable $s$ is in the vicinity of the complex pole position,

$$
z_{\Delta}=m_{\Delta}-i \frac{\Gamma_{\Delta}}{2}
$$

the process is dominated by the propagation of a $\Delta$ resonance in the $s$ channel (see Fig. 1). Since the $W$ boson induces the transition between the nucleon and the delta in terms of the $V-A$ structure of the coupling to the quarks, this contribution is sensitive to both the nucleon-to-delta vector and axial-vector transitions. One now parametrizes the contribution of the unstable $\Delta(1232)$ and defines the form factors in analogy to a stable particle. For an unstable particle such as the $\Delta(1232)$, "on-shell kinematics" are given by the complex pole position. The extraction of the transition form factors from weak pion-production data in the delta-resonance region could, in principle, be performed in analogy to the determination of the electromagnetic transition form factors from pion electroproduction data using a partial wave analysis [15].

\section{B. Definition of the axial-vector transition form factors}

In the following, we provide a short definition of the form factors. We stick to the notation of Ref. [32], where more details can be found. In terms of the light-quark field operators, $q=(u, d)^{T}$, the Cartesian components of the isovector axial-vector current operator are given by [49]

$$
A_{j}^{\mu}(x)=\bar{q}(x) \gamma^{\mu} \gamma_{5} \frac{\tau_{j}}{2} q(x) .
$$

\footnotetext{
${ }^{3}$ From a theoretical point of view, it is possible to study a hypothetical situation, where the sum of the nucleon and pion masses is larger than the $\Delta$ mass, resulting in a stable $\Delta$ state.
}

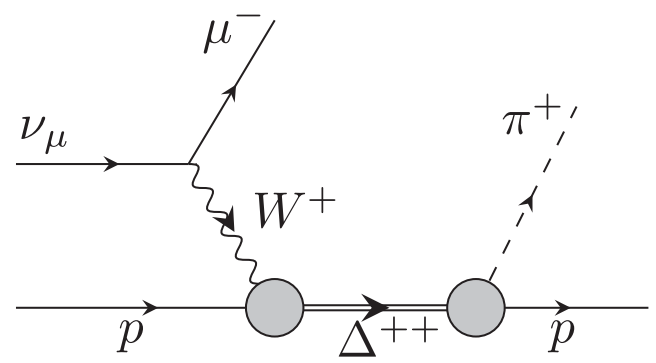

FIG. 1. At $s \approx m_{\Delta}^{2}$, the process is dominated by the $s$-channel pole diagram due to the propagator of the $\Delta(1232)$. The nucleon, electron, and neutrino are represented by single lines, the $\Delta(1232)$ by a double line, the $W$ boson by a wiggly line, and the pion by a dashed line. The circles represent dressed vertices.

In general, the invariant amplitude ${ }^{4}$ for a transition between hadronic states $\left|A\left(p_{i}\right)\right\rangle$ and $\left|B\left(p_{f}\right)\right\rangle$, induced by a planewave external field of the form $a_{\mu, j}(x)=\epsilon_{\mu, j}(q) e^{-i q \cdot x}$, is defined as

$$
\mathcal{M}=i \epsilon_{\mu, j}(q)\left\langle B\left(p_{f}\right)\left|A_{j}^{\mu}(0)\right| A\left(p_{i}\right)\right\rangle,
$$

where four-momentum conservation $p_{f}=p_{i}+q$ due to translational invariance is implied.

Introducing the spherical tensor notation [50],

$$
A_{ \pm 1}^{\mu(1)}=\mp \frac{1}{\sqrt{2}}\left(A_{1}^{\mu} \pm i A_{2}^{\mu}\right), \quad A_{0}^{\mu(1)}=A_{3}^{\mu},
$$

and using isospin symmetry, we express the matrix element of the spherical isospin components $(\alpha=+1,0,-1)$ between a nucleon state and a $\Delta$ state as

$$
\begin{aligned}
& \left\langle 3 / 2, \tau_{\Delta}\left|A_{\alpha}^{\mu(1)}\right| 1 / 2, \tau\right\rangle \\
& \quad=\left(1 / 2, \tau ; 1, \alpha \mid 3 / 2, \tau_{\Delta}\right)\left\langle 3 / 2|| A^{\mu(1)}|| 1 / 2\right\rangle,
\end{aligned}
$$

where $\left\langle 3 / 2|| A^{\mu(1)} \| 1 / 2\right\rangle$ denotes the reduced matrix element and $\left(1 / 2, \tau ; 1, \alpha \mid 3 / 2, \tau_{\Delta}\right)$ is the relevant Clebsch-Gordan coefficient. The reduced matrix element may, for example, be obtained from the $p$ to $\Delta^{+}$transition,

$$
\left\langle 3 / 2|| A^{\mu(1)}|| 1 / 2\right\rangle=\sqrt{\frac{3}{2}}\left\langle\Delta^{+}\left|A_{0}^{\mu(1)}\right| p\right\rangle .
$$

The Lorentz structure of the reduced matrix element may be written as

$$
\left\langle\Delta\left(p_{f}, s_{f}\right)\left\|A^{\mu(1)}(0)\right\| N\left(p_{i}, s_{i}\right)\right\rangle=\bar{w}_{\lambda}\left(p_{f}, s_{f}\right) \Gamma_{A}^{\lambda \mu} u\left(p_{i}, s_{i}\right) .
$$

\footnotetext{
${ }^{4}$ Our convention for the invariant amplitude complies with Ref. [47]. In particular, it contains the imaginary unit on the righthand side of Eq. (2).
} 
Here, the initial nucleon is described by the Dirac spinor $u\left(p_{i}, s_{i}\right)$ with a real mass $m_{N}$ and $p_{i}^{2}=m_{N}^{2}$, the final $\Delta(1232)$ is described via the Rarita-Schwinger vectorspinor $\bar{w}_{\lambda}\left(p_{f}, s_{f}\right)[51,52]$ with a complex mass $z_{\Delta}$ and $p_{f}^{2}=z_{\Delta}^{2}[38,53]$. In the following, it is always understood that the "tensor" $\Gamma_{A}^{\lambda \mu}$ is evaluated between on-shell spinors $u$ and $\bar{w}_{\lambda}$, satisfying ${ }^{5}$

$$
\begin{aligned}
\not p_{i} u\left(p_{i}, s_{i}\right) & =m_{N} u\left(p_{i}, s_{i}\right), \\
\bar{w}_{\lambda}\left(p_{f}, s_{f}\right) \not p_{f} & =z_{\Delta} \bar{w}_{\lambda}\left(p_{f}, s_{f}\right), \quad \bar{w}_{\lambda}\left(p_{f}, s_{f}\right) \gamma^{\lambda}=0, \\
\bar{w}_{\lambda}\left(p_{f}, s_{f}\right) p_{f}^{\lambda} & =0 .
\end{aligned}
$$

The expressions for a stable $\Delta$ resonance are obtained via the replacement $z_{\Delta} \rightarrow m_{\Delta}$. The "tensor" $\Gamma_{A}^{\lambda \mu}$ contains a superposition of four Lorentz tensors [48,54], which we choose as $[31,34]$

$$
\begin{aligned}
\Gamma_{A}^{\lambda \mu}= & \frac{C_{3}^{A}\left(Q^{2}\right)}{m_{N}}\left(g^{\lambda \mu} \phi q-q^{\lambda} \gamma^{\mu}\right)+\frac{C_{4}^{A}\left(Q^{2}\right)}{m_{N}^{2}}\left(g^{\lambda \mu} p_{f} \cdot q-q^{\lambda} p_{f}^{\mu}\right) \\
& +C_{5}^{A}\left(Q^{2}\right) g^{\lambda \mu}+\frac{C_{6}^{A}\left(Q^{2}\right)}{m_{N}^{2}} q^{\lambda} q^{\mu},
\end{aligned}
$$

where $Q^{2}=-q^{2}$. Note that $p_{f} \cdot q=\frac{1}{2}\left(p_{f}+p_{i}+p_{f}-p_{i}\right)$ $\cdot\left(p_{f}-p_{i}\right)=\frac{1}{2}\left(p_{f}^{2}-p_{i}^{2}+q^{2}\right)=\frac{1}{2}\left(z_{\Delta}^{2}-m_{N}^{2}-Q^{2}\right)$. Our sign convention for the form factors in Eq. (7) is such that we parameterize the matrix element of $+A_{j}^{\mu}$ and, thus, our sign convention follows closely the convention of the nucleon-to-nucleon axial-vector transition [55]. In particular, $C_{5}^{A}\left(Q^{2}\right)$ and $C_{6}^{A}\left(Q^{2}\right)$ correspond to the axial nucleon form factor $G_{A}\left(Q^{2}\right)$ and the induced pseudoscalar form factor $G_{P}\left(Q^{2}\right)$, respectively.

\section{Pion-nucleon-delta transition form factor}

Assuming isospin symmetry, i.e., equal up-quark and down-quark masses $m_{u}=m_{d}=\hat{m}$, the divergence of the axial-vector current is given by $[55,56]$

$$
\partial_{\mu} A_{j}^{\mu}(x)=\hat{m} P_{j}(x)
$$

where

$$
P_{j}(x)=i \bar{q}(x) \gamma_{5} \tau_{j} q(x)
$$

denotes the pseudoscalar density [49]. With the help of the pion mass $M_{\pi}$ and the pion-decay constant $F_{\pi}$, the isovector operator $\Phi_{j}(x) \equiv \hat{m} P_{j}(x) /\left(M_{\pi}^{2} F_{\pi}\right)$ serves as an interpolating pion field [56] such that Eq. (8) amounts to the standard PCAC relation (partially conserved axialvector current) [57]. By means of $\Phi_{j}(x)$ we define the $\pi N \Delta$ transition form factor $G_{\pi N \Delta}\left(Q^{2}\right)$ in analogy to the $\pi N$ form factor $G_{\pi N}\left(Q^{2}\right)[55,58]$ as [34]

\footnotetext{
${ }^{5}$ The explicit form of $\bar{w}_{\lambda}$ can be found in Ref. [53].
}

$$
\begin{aligned}
& \left\langle\Delta\left(p_{f}, s_{f}\right)\left\|\Phi^{(1)}(0)\right\| N\left(p_{i}, s_{i}\right)\right\rangle \\
& \quad=i \frac{1}{M_{\pi}^{2}+Q^{2}} G_{\pi N \Delta}\left(Q^{2}\right) \bar{w}_{\lambda}\left(p_{f}, s_{f}\right) \frac{q^{\lambda}}{m_{N}} u\left(p_{i}, s_{i}\right) .
\end{aligned}
$$

From Eq. (8) we obtain

$$
\begin{aligned}
& i q_{\mu}\left\langle\Delta\left(p_{f}, s_{f}\right)\left\|A^{\mu(1)}(0)\right\| N\left(p_{i}, s_{i}\right)\right\rangle \\
& =\hat{m}\left\langle\Delta\left(p_{f}, s_{f}\right)\left\|P^{(1)}(0)\right\| N\left(p_{i}, s_{i}\right)\right\rangle,
\end{aligned}
$$

which, using Eqs. (7) and (10), results in

$G_{\pi N \Delta}\left(Q^{2}\right)=\frac{m_{N}}{F_{\pi}} \frac{M_{\pi}^{2}+Q^{2}}{M_{\pi}^{2}}\left[C_{5}^{A}\left(Q^{2}\right)-\frac{Q^{2}}{m_{N}^{2}} C_{6}^{A}\left(Q^{2}\right)\right]$.

In other words, once we know the form factors $C_{5}^{A}\left(Q^{2}\right)$ and $C_{6}^{A}\left(Q^{2}\right)$, we can also extract the form factor $G_{\pi N \Delta}\left(Q^{2}\right)$. The $\pi N \Delta$ coupling constant $g_{\pi N \Delta}$ is defined as

$$
g_{\pi N \Delta}=G_{\pi N \Delta}\left(-M_{\pi}^{2}\right) .
$$

Since the form factor $C_{6}^{A}\left(Q^{2}\right)$ has a pole at $Q^{2}=-M_{\pi}^{2}$, the coupling constant $g_{\pi N \Delta}$ does not vanish despite the factor $\left(M_{\pi}^{2}+Q^{2}\right)$ in Eq. (12).

\section{EFFECTIVE LAGRANGIAN AND POWER COUNTING}

In this section, we provide the interaction Lagrangians relevant for the calculation of the isovector axial-vectorcurrent form factors of the nucleon-to-delta transition in covariant chiral EFT. The effective Lagrangian, $\mathcal{L}_{\text {eff }}$, consists of a purely pionic, a pion-nucleon, a pion-delta, and a pion-nucleon-delta Lagrangian, each of which is organized in a combined derivative and quark-mass expansion. The most general effective Lagrangian for the calculation of the transition form factors up to and including order $q^{3}$ is given by

$$
\begin{aligned}
\mathcal{L}_{\text {eff }}= & \mathcal{L}_{\pi}^{(2)}+\mathcal{L}_{\pi}^{(4)}+\mathcal{L}_{\pi N}^{(1)}+\mathcal{L}_{\pi \Delta}^{(1)} \\
& +\mathcal{L}_{\pi N \Delta}^{(1)}+\mathcal{L}_{\pi N \Delta}^{(2)}+\mathcal{L}_{\pi N \Delta}^{(3)}+\ldots,
\end{aligned}
$$

where the ellipsis denotes terms which are either of higher order or irrelevant for our calculation.

The perturbative calculation of the transition matrix element is organized by applying the "standard" power counting to the renormalized diagrams (see, e.g., Ref. [59]), i.e., an interaction vertex obtained from an $\mathcal{O}\left(q^{n}\right)$ Lagrangian counts as order $q^{n}$, a pion propagator as order $q^{-2}$, a nucleon propagator as order $q^{-1}$, and the integration of a loop as order $q^{4}$. We assign the order $q^{-1}$ to the $\Delta$ propagator if it appears in loop integrals. ${ }^{6}$ Also the order $q^{1}$ is assigned to the leading

\footnotetext{
${ }^{6}$ In resonance generating channels, a $\Delta$ propagator which is not involved in a loop integration has to be dressed. In this case, one re-sums the self-energy insertions and considers the dressed propagator as of order $q^{-3}$, because the self-energy starts at $\mathcal{O}\left(q^{3}\right)$ [39].
} 
order of the mass difference $m_{\Delta}-m_{N}$. In other words, we apply the so-called small-scale expansion of Ref. [60]. When calculating the form factors of Eqs. (7) and (10) at the oneloop level, we follow the strategy of keeping all $Q^{2}$ dependent terms, even if they are of higher order when applying an additional expansion with respect to a heavy mass such as the nucleon mass or the delta mass.

The pionic Lagrangians at $\mathcal{O}\left(q^{2}\right)$ and $\mathcal{O}\left(q^{4}\right)$ are given by $[49,56]$

$$
\begin{aligned}
& \mathcal{L}_{\pi}^{(2)}=\frac{F^{2}}{4}\left\{\operatorname{Tr}\left[D_{\mu} U\left(D^{\mu} U\right)^{\dagger}\right]+\operatorname{Tr}\left(\chi U^{\dagger}+U \chi^{\dagger}\right)\right\}, \\
& \left.\mathcal{L}_{\pi}^{(4)}=\frac{l_{4}}{4} \operatorname{Tr}\left[D_{\mu} U\left(D^{\mu} \chi\right)^{\dagger}\right]+D_{\mu} \chi\left(D^{\mu} U\right)^{\dagger}\right]+\ldots,
\end{aligned}
$$

respectively, with $\chi=2 B(s+i p), s$ and $p$ denoting the scalar and pseudoscalar external sources [49]. $F$ is the piondecay constant in the chiral limit, $F_{\pi}=F[1+\mathcal{O}(\hat{m})]=$ $92.2 \mathrm{MeV}$, and $B$ is associated with the scalar singlet quark condensate $\langle\bar{q} q\rangle_{0}$ in the chiral limit $[49,56,61]$. We employ $\mathrm{SU}(2)$ isospin symmetry, $m_{u}=m_{d}=\hat{m}$, and the lowestorder prediction for the pion mass squared is $M^{2}=2 B \hat{m}$ $[49,56]$, resulting from inserting the quark masses into the external scalar field, $s=\hat{m} \mathbb{1}$. The triplet of pion fields is contained in the unimodular, unitary, $(2 \times 2)$ matrix $U$,

$U=u^{2}=\exp \left(i \frac{\Phi}{F}\right), \Phi=\tau_{j} \phi_{j}=\left(\begin{array}{cc}\pi^{0} & \sqrt{2} \pi^{+} \\ \sqrt{2} \pi^{-} & -\pi^{0}\end{array}\right)$,

where $\tau_{j}$ are the Pauli matrices. Introducing external vector fields $v_{\mu}$ and axial-vector fields $a_{\mu}$ as

$$
v_{\mu}=\frac{\tau_{j}}{2} v_{\mu, j}, \quad a_{\mu}=\frac{\tau_{j}}{2} a_{\mu, j},
$$

and using

$$
r_{\mu}=v_{\mu}+a_{\mu}, \quad l_{\mu}=v_{\mu}-a_{\mu},
$$

the covariant derivative of $U$ is defined as

$$
D_{\mu} U=\partial_{\mu} U-i r_{\mu} U+i U l_{\mu} .
$$

The leading-order pion-nucleon Lagrangian reads $[56,58]$

$\mathcal{L}_{\pi N}^{(1)}=\bar{\Psi}\left(i \not D-m+\frac{g_{A}}{2} \gamma^{\mu} \gamma_{5} u_{\mu}\right) \Psi, \quad \Psi=\left(\begin{array}{l}p \\ n\end{array}\right)$,

where $\Psi$ denotes the nucleon isospin doublet containing the four-component Dirac fields for the proton and the neutron. The covariant derivative $D_{\mu} \Psi$ is given by ${ }^{7}$

\footnotetext{
${ }^{7}$ We do not consider a coupling to an external isoscalar vector field.
}

$$
\begin{aligned}
D_{\mu} \Psi & =\left(\partial_{\mu}+\Gamma_{\mu}\right) \Psi, \\
\Gamma_{\mu} & =\frac{1}{2}\left[u^{\dagger}\left(\partial_{\mu}-i r_{\mu}\right) u+u\left(\partial_{\mu}-i l_{\mu}\right) u^{\dagger}\right] .
\end{aligned}
$$

The chiral vielbein is defined as

$$
u_{\mu}=u_{\mu, j} \tau_{j}=i\left[u^{\dagger}\left(\partial_{\mu}-i r_{\mu}\right) u-u\left(\partial_{\mu}-i l_{\mu}\right) u^{\dagger}\right]
$$

The Lagrangian $\mathcal{L}_{\pi N}^{(1)}$ contains two free parameters, namely, the nucleon mass in the chiral limit, $m$, and the axial-vector coupling constant in the chiral limit, $g_{A}$. Expanding $u_{\mu, j}$ as

$$
u_{j, \mu}=a_{\mu, j}-\frac{\partial_{\mu} \phi_{j}}{F}+\mathcal{O}\left(v_{\mu} \Phi, a_{\mu} \Phi^{2}, \partial_{\mu} \Phi \Phi^{2}\right),
$$

Eq. (20) gives rise to the lowest-order $\pi N N$ vertex as well as the axial-vector $N N$ transition vertex which are both needed for the one-loop corrections at $\mathcal{O}\left(q^{3}\right)$.

The building blocks for constructing the Lagrangian of the $\Delta$ resonance can be found in Refs. [39,59] and the references therein. For our purposes, we only need the leading-order contribution, ${ }^{8}$

$$
\begin{aligned}
\mathcal{L}_{\pi \Delta}^{(1)}= & -\bar{\Psi}_{\mu} \xi^{\frac{3}{2}}\left[\left(i \not D-m_{\Delta}\right) g^{\mu \nu}-i\left(\gamma^{\mu} D^{\nu}+\gamma^{\nu} D^{\mu}\right)+i \gamma^{\mu} \not D \gamma^{\nu}\right. \\
& +m_{\Delta} \gamma^{\mu} \gamma^{\nu}+\frac{g_{1}}{2}\left(\not h \gamma_{5} g^{\mu \nu}-\gamma^{\mu} u^{\nu} \gamma_{5}-u^{\mu} \gamma^{\nu} \gamma_{5}\right. \\
& \left.\left.-\gamma^{\mu} \not l \gamma_{5} \gamma^{\nu}\right)\right] \xi^{\frac{3}{2}} \Psi_{\nu}
\end{aligned}
$$

from the $\pi \Delta$ Lagrangian, where $\Psi_{\nu}$ denotes a vector-spinor isovector-isospinor field. The isovector-isospinor transforms under the $1 \otimes \frac{1}{2}=\frac{3}{2} \oplus \frac{1}{2}$ representation and, thus, contains both isospin $3 / 2$ and isospin $1 / 2$ components. In order to describe the $\Delta$, it is necessary to project onto the isospin- $3 / 2$ subspace. The corresponding matrix representation of the projection operator is denoted by $\xi^{\frac{3}{2}}$, and the entries of $\xi^{\frac{3}{2}}$ are given by [59]

$$
\xi_{i j}^{\frac{3}{2}}=\delta_{i j}-\frac{1}{3} \tau_{i} \tau_{j}
$$

Inserting the expansion of Eq. (23) into Eq. (24), we obtain the leading-order $\pi \Delta \Delta$ vertex which is proportional to $g_{1}$ and which is needed for the one-loop corrections at $\mathcal{O}\left(q^{3}\right)$.

The leading-order $\pi N \Delta$ chiral Lagrangian is given by [see Eq. (4.200) of Ref. [59] with $\tilde{z}=-1$ for consistency with the choice $A=-1$ ]

$$
\mathcal{L}_{\pi N \Delta}^{(1)}=g \bar{\Psi}_{\lambda, i} \xi_{i j}^{\frac{3}{2}}\left(g^{\lambda \mu}-\gamma^{\lambda} \gamma^{\mu}\right) u_{\mu, j} \Psi+\text { H.c. },
$$

where H.c. denotes the Hermitian conjugate. Expanding $u_{\mu, j}$ as above, Eq. (25) gives rise to the leading-order

\footnotetext{
${ }^{8}$ Note that the free Lagrangian contains an arbitrary real parameter $A \neq-\frac{1}{2}[62,63]$, for which we choose $A=-1$ such that the propagator takes the simplest form.
} 
contribution to $C_{5}^{A}\left(Q^{2}\right)$ as well as the leading-order $\pi N \Delta$ vertex, which is needed for the calculation of the loop contributions at $\mathcal{O}\left(q^{3}\right)$.

At $\mathcal{O}\left(q^{3}\right)$, the higher-order Lagrangians $\mathcal{L}_{\pi N \Delta}^{(2)}$ and $\mathcal{L}_{\pi N \Delta}^{(3)}$ can only contribute at the tree level. In principle, these Lagrangians were derived in Ref. [64] (see also Ref. [65]). Taking Eq. (66) of Ref. [64] for $\mathcal{L}_{\pi N \Delta}^{(2)}$, there would be no contribution to the form factors at $\mathcal{O}\left(q^{2}\right)$, because the first two terms contain the chiral vielbein quadratically, and the last term involves the "wrong" field strength tensor $f_{+}^{\mu \nu}$. However, as discussed in Appendix, there are independent contributions at $\mathcal{O}\left(q^{2}\right)$ and $\mathcal{O}\left(q^{3}\right)$. In fact, this is to be expected for the following reason. Counting the polarization vector as of $\mathcal{O}(q)$ and treating only the four-momentum $q$ (but not $p_{f}$ ) as a small quantity, we expect from Eqs. (4) and (7) two free parameters related to $C_{3}^{A}(0)$ and $C_{4}^{A}(0) .{ }^{9}$ For the sake of simplicity, we will denote these two parameters by $\alpha$ and $\beta$, respectively (see Appendix), and will refer to them as of beyond leading order. Similarly, the $\mathcal{O}\left(q^{3}\right)$ Lagrangian of Ref. [64] produces fewer contributions to the form factors than is expected from the counting of momenta (and the polarization vector). Since it is not the purpose of this paper to construct the most general Lagrangian at $\mathcal{O}\left(q^{3}\right)$, we have decided to Taylor expand the form factors and keep the expansion coefficients as free parameters.

\section{RESULTS}

Figure 2 shows those tree-level and one-loop Feynman diagrams that generate a nonvanishing contribution to the nucleon-to-delta transition matrix element of the isovector axial-vector current. In principle, the renormalized vertex is obtained by multiplying the contributions of Fig. 2 by the square roots of the wave function renormalization constants $Z_{N}$ and $Z_{\Delta}$. In practice, we evaluate the loop diagrams in the framework of dimensional regularization at the renormalization scale $\mu=1 \mathrm{GeV}$. We apply the modified minimal subtraction scheme of ChPT (MS) [49,59] by dropping infinite parts in terms of the combination $2 /(n-4)-$ $\left[\ln \left(4 \pi+\Gamma^{\prime}(1)+1\right)\right]$, where $n$ denotes the number of space-time dimensions. We combine the remaining finite pieces with the available renormalized free parameters. When calculating the loop contributions involving a delta line in the loop [diagrams (n), (p), (q), (r), (s), (t), and (u) of Fig. 2], we neglect the width. This amounts to neglecting terms of $\mathcal{O}\left(\hbar^{2}\right)$, which are beyond the accuracy of a oneloop calculation.

\footnotetext{
${ }^{9}$ In an effective Lagrangian, the four-momentum $p_{f}$ of the Lorentz structure multiplying $C_{4}^{A}\left(Q^{2}\right)$ would result from a (hard) derivative, acting on the delta field. Using a total derivative, the equations of motion, and the constraint equations for the delta field [essentially Eqs. (5) and (6)], such a term can be related to a Lagrangian of $\mathcal{O}\left(q^{3}\right)$ without a hard derivative.
}

In fact, for the actual calculation, we use a decomposition of $\Gamma_{A}^{\lambda \mu}$ which differs from Eq. (7), namely,

$$
\Gamma_{A}^{\lambda \mu}=k_{1} q^{\lambda} p_{f}^{\mu}+k_{2} q^{\lambda} q^{\mu}+k_{3} q^{\lambda} \gamma^{\mu}+k_{4} g^{\lambda \mu} .
$$

For each diagram, we extract the four coefficients $k_{i}$ and determine their contributions to the form factors $C_{i}^{A}\left(Q^{2}\right)$, using the relations

$$
\begin{aligned}
& C_{3}^{A}=-m_{N} k_{3}, \\
& C_{4}^{A}=-m_{N}^{2} k_{1}, \\
& C_{5}^{A}=\frac{1}{2}\left(z_{\Delta}^{2}-m_{N}^{2}-Q^{2}\right) k_{1}+\left(z_{\Delta}-m_{N}\right) k_{3}+k_{4}, \\
& C_{6}^{A}=m_{N}^{2} k_{2} .
\end{aligned}
$$

Using the strategy outlined in Sec. III, the tree-level contributions to the form factors can be written as ${ }^{10}$

$$
\begin{aligned}
& C_{3 \text { tree }}^{A}\left(Q^{2}\right)=\alpha, \\
& C_{4 \text { tree }}^{A}\left(Q^{2}\right)=\beta, \\
& C_{5 \text { tree }}^{A}\left(Q^{2}\right)=\mathrm{g}+\gamma M^{2}+\delta Q^{2}, \\
& C_{6 \text { tree }}^{A}\left(Q^{2}\right)=m_{N}^{2} \frac{\mathrm{g}+\gamma M^{2}+\epsilon M^{2}}{M^{2}+Q^{2}}+\zeta .
\end{aligned}
$$

We have explicitly shown the quark-mass dependence in terms of the lowest-order squared pion mass $M^{2}{ }^{11}$ In addition, we made use of the analogy of the form factors $C_{5}^{A}\left(Q^{2}\right)$ and $C_{6}^{A}\left(Q^{2}\right)$ to the nucleon form factors $G_{A}\left(Q^{2}\right)$ and $G_{P}\left(Q^{2}\right)$ (see Ref. [55]). At leading and nextto-leading order, the form factors $C_{3}^{A}\left(Q^{2}\right), C_{4}^{A}\left(Q^{2}\right)$, and $C_{5}^{A}\left(Q^{2}\right)$ are constant. The parameters g, $\alpha$, and $\beta$ are independent low-energy constants, i.e., they are not predicted by chiral symmetry. Using the relation from the static quark model with $\mathrm{SU}(6)$ symmetry results in the estimate $\mathrm{g}=3 \sqrt{2} \mathrm{~g}_{A} / 5[32,60]$. At this order, the form factor $C_{6}^{A}\left(Q^{2}\right)$ is predicted as

$$
C_{6}^{A}\left(Q^{2}\right)=\frac{m_{N}^{2}}{M^{2}+Q^{2}} g .
$$

Turning to $\mathcal{O}\left(q^{3}\right)$, we will now have both treelevel modifications as well as loop contributions. The parameter $\gamma$ corresponds to a quark-mass correction to the

\footnotetext{
${ }^{10}$ For the sake of simplicity, we do not display the contribution of diagrams (e) and (g) to the formula for $C_{6 \text { tree }}^{A}\left(Q^{2}\right)$. After renormalization, the diagrams $(\mathrm{d}),(\mathrm{e}),(\mathrm{g}),(\mathrm{j})$, and $(\mathrm{k})$ add up such that a factor $F /\left(Q^{2}+M^{2}\right)$ is replaced by $F_{\pi} /\left(Q^{2}+M_{\pi}^{2}\right)$.

${ }^{11}$ In Ref. [66], a different organization scheme-the so-called $\delta$ expansion-was proposed for the effective field theory including the delta resonance. According to Ref. [30], the use of the $\delta$ expansion in the calculation of the axial-vector $N \Delta$ transition form factors amounts to shifting explicit $M_{\pi}^{2}$ corrections to higher orders. Using footnote 4 of Ref. [30], these higher-order terms correspond to our terms proportional to $\gamma$ and $\epsilon$.
} 


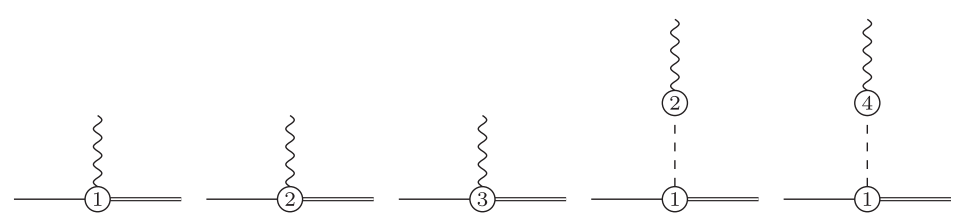

(a)

(b)

(c)

(d)

(e)

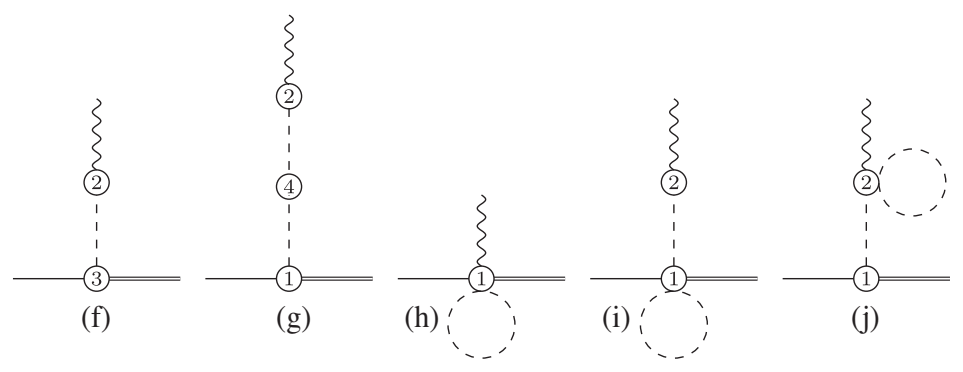

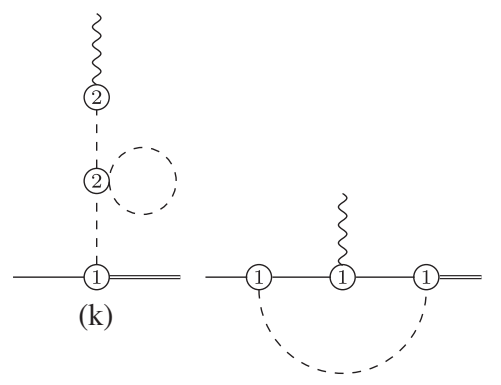

(1)

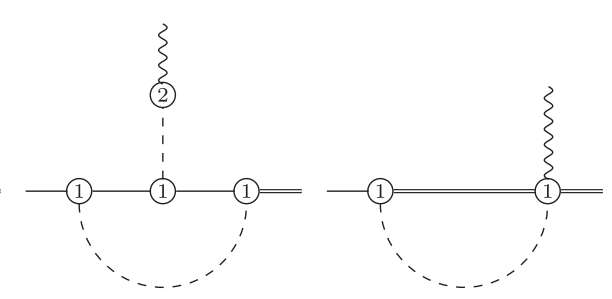

(n)

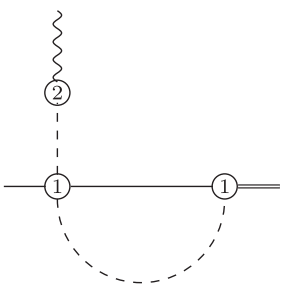

(o)

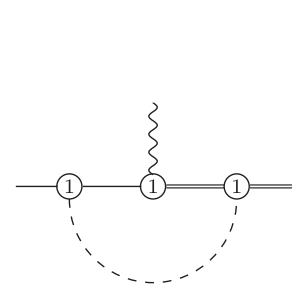

(p) (m)

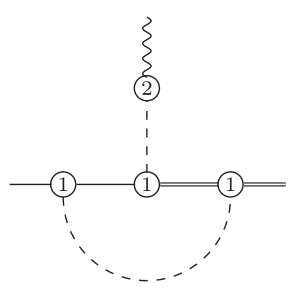

(q)

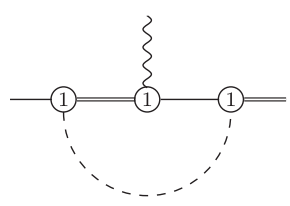

(r)

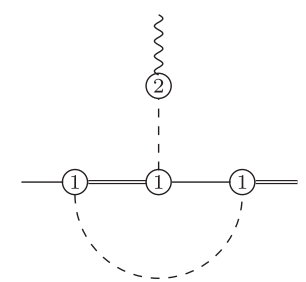

(s)

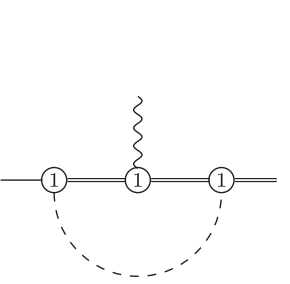

(t)

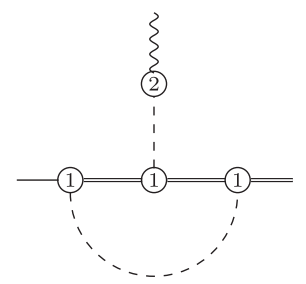

(u)

FIG. 2. Diagrams which, after renormalization, result in nonvanishing contributions to the axial-vector nucleon-to-delta transition form factors up to and including $\mathcal{O}\left(q^{3}\right)$. The double, solid, dashed, and wiggly lines correspond to the delta, nucleon, pion, and the external axial-vector source, respectively.

nucleon-to-delta transition axial-vector coupling constant $g_{A N \Delta} \equiv C_{5}^{A}(0)$. Furthermore, $\delta$ contributes to the meansquare axial transition radius

$$
\left\langle r_{A N \Delta}^{2}\right\rangle \equiv-\left.\frac{6}{g_{A N \Delta}} \frac{d C_{5}^{A}\left(Q^{2}\right)}{d Q^{2}}\right|_{Q^{2}=0} .
$$

The parameter $\epsilon$ enters into the calculation of the generalized Goldberger-Treiman discrepancy [67-69]

$$
\Delta \equiv 1-\frac{m_{N} g_{A N \Delta}}{F_{\pi} g_{\pi N \Delta}},
$$

where $g_{\pi N \Delta}$ is defined in Eq. (13). Finally, the parameter $\zeta$ is related to the $y$-intercept of $C_{6}^{A}\left(Q^{2}\right)$.

Unfortunately, there is very little empirical information on the form factors $C_{i}^{A}\left(Q^{2}\right)$. In order to obtain some estimate about the parameters and shape of our theoretical results, we perform a fit to the parametrization 


$$
C_{i}^{A}\left(Q^{2}\right)=\frac{C_{i}^{A}(0)\left(1+a_{i} \frac{Q^{2}}{b_{i}+Q^{2}}\right)}{\left(1+\frac{Q^{2}}{M_{A}^{2}}\right)^{2}} \quad(i=3,4,5)
$$

(see Appendix D of Ref. [70]). The form factor $C_{6}^{A}\left(Q^{2}\right)$ is assumed to be dominated by the pion-pole contribution. In particular, we make use of the axial-vector form factor parameters of the Adler model (see Table D.1 of Ref. [70]),

$$
\begin{aligned}
& C_{3}^{A}(0)=0, \quad a_{3}=0, \quad b_{3}=0, \\
& C_{4}^{A}(0)=-0.3, \quad a_{4}=-1.21, \quad b_{4}=2.0 \mathrm{GeV}^{2}, \\
& C_{5}^{A}(0)=1.2, \quad a_{5}=-1.21, \quad b_{5}=2.0 \mathrm{GeV}^{2} .
\end{aligned}
$$

This parametrization was used by the authors of Ref. [21] to extract the axial mass as $M_{A}=\left(1.28_{-0.10}^{+0.08}\right) \mathrm{GeV}$ from their analysis of the $\Delta^{++}$production reaction $\nu_{\mu}+d \rightarrow \mu^{-}+$ $\Delta^{++}+n_{s}$, where $n_{s}$ refers to a spectator neutron. Note that the parameters of Eq. (31) were used in Ref. [21] without any uncertainty such that the only uncertainty in the following input to out fits will be the uncertainty of $M_{A}$.

To emphasize the low- $Q^{2}$ region, we choose the $Q^{2}$ values at which to evaluate the empirical form factors according to $Q_{n}^{2}=n^{2} \times 0.0004 \mathrm{GeV}^{2}(n=0,1, \ldots, 28)$. For the fits, we employ the Mathematica routine NonlinearModelFit. Our loop diagrams contain the lowenergy constants (LECs) $g_{A}, g_{1}$, and $g$. In the loop integrals, we approximate $g_{A}$ by $g_{A}=1.28$ (empirical value $g_{A}=1.2756 \pm 0.0013[1]$ ), because the difference between $g_{A}$ and $g_{A}$ is of order $M^{2}$ in the chiral expansion and, therefore, using $g_{A}$ introduces an error of higher order beyond the accuracy of our calculation. For the other two coupling constants, we make use of an SU(6) spin-flavor quark-model relation $[59,60]$,

$$
\mathrm{g}_{1}=\frac{9}{5} \mathrm{~g}_{A}, \quad \mathrm{~g}=\frac{3}{5} \sqrt{2} \mathrm{~g}_{A},
$$

resulting in $g_{1}=2.30$ and $g=1.08$, respectively. ${ }^{12}$ Furthermore, we replace $M^{2}$ by $M_{\pi}^{2}$ and use $M_{\pi}=$ $0.135 \mathrm{GeV}$. From a fit to the form factors $C_{4}^{A}\left(Q^{2}\right)$ and $C_{5}^{A}\left(Q^{2}\right)$, we obtain for the LECs $\beta, \gamma$, and $\delta$ the values

$\beta=0.335_{-0.003}^{+0.004}, \quad \gamma=-84.7_{-0.1}^{+0.2} \mathrm{GeV}^{-2}$,

$\delta=-3.20_{-0.09}^{+0.14} \mathrm{GeV}^{-2}$.

The value $C_{5}^{A}(0)$ is the analogue of $g_{A}$. The individual contributions to $C_{5}^{A}(0)$ are given by ${ }^{13}$

\footnotetext{
${ }^{12}$ Using the leading-order term of Eq. (25) only to describe the width, would result in $\mathrm{g}=1.13$ [71].

${ }^{13}$ In the following, we give our numerical results after rounding with three significant digits.
}

$$
\begin{aligned}
C_{5}^{A}(0) & =1.20_{-0.01}^{+0.00}=\mathrm{g}+\gamma M_{\pi}^{2}+\text { loops } \\
& =1.08-1.54_{-0.01}^{+0.00}+1.66 .
\end{aligned}
$$

The $M_{\pi}^{2}$ term plus the loops amount to a $10 \%$ correction of the leading-order term. The uncertainty of $C_{5}^{A}(0)$ in Eq. (34) results entirely from the uncertainty of $\gamma$, which in turn results from the value of the axial mass $M_{A}$ used in the empirical parametrization. This should not be confused with an estimate of higher-order corrections to be expected from applying effective field theory beyond $\mathcal{O}\left(q^{3}\right)$. We will come back to a qualitative estimate of the importance of higher-order terms at the beginning of Sec. V.

Varying exclusively the parameter $M_{A}$ in the empirical parametrization, we do not expect to obtain a reliable estimate in the uncertainty of the empirical form factors. Therefore, except for two illustrative examples (see below), we will refrain from showing error bands, as they might suggest a greater degree of accuracy than is actually available. In Fig. 3(a), we display the individual contributions to $C_{5}^{A}\left(Q^{2}\right)$ for $\mathrm{g}_{1}=2.30$; the total result is given by the red solid line, the loop contribution by the green dashed line, and the tree-level contribution by the green dotted line. The total result shows, as was to be expected from a calculation at $\mathcal{O}\left(q^{3}\right)$, essentially a linear behavior as a function of $Q^{2}$. The loop contribution rises with increasing $Q^{2}$, whereas the tree-level contribution decreases linearly with $Q^{2}$. A comparison with the empirical parametrization of the Adler model is shown in Fig. 4. The pink error band of our calculation overlaps the narrower gray error band of the empirical parametrization. In the $Q^{2}$ range considered, the fit differs by less than $3 \%$ from the empirical parametrization.

Using Eq. (28), we obtain for the mean-square axial transition radius

$$
\left\langle r_{A N \Delta}^{2}\right\rangle=0.345 \mathrm{fm}^{2}
$$

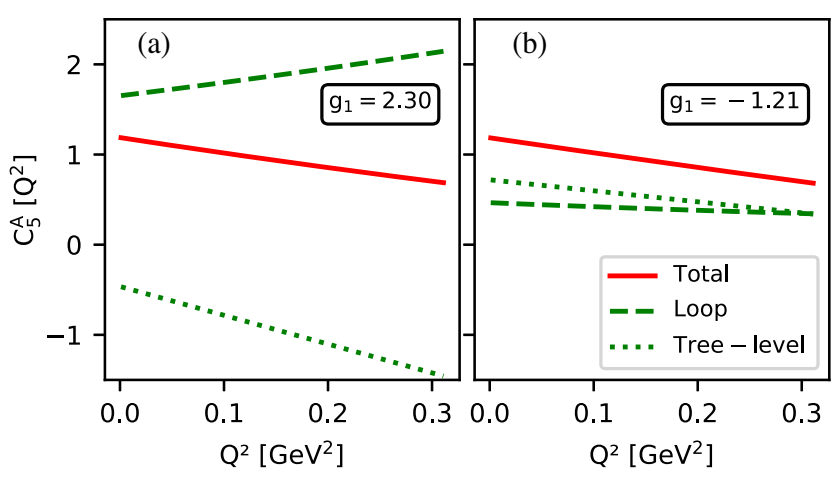

FIG. 3. Individual contributions to $C_{5}^{A}\left(Q^{2}\right)$. The red solid, green dotted, and green dashed lines correspond to the total result, the loop contribution, and the tree-level contribution, respectively. The left and right panels show the fits for $g_{1}=2.30$ and $g_{1}=-1.21$, respectively. 


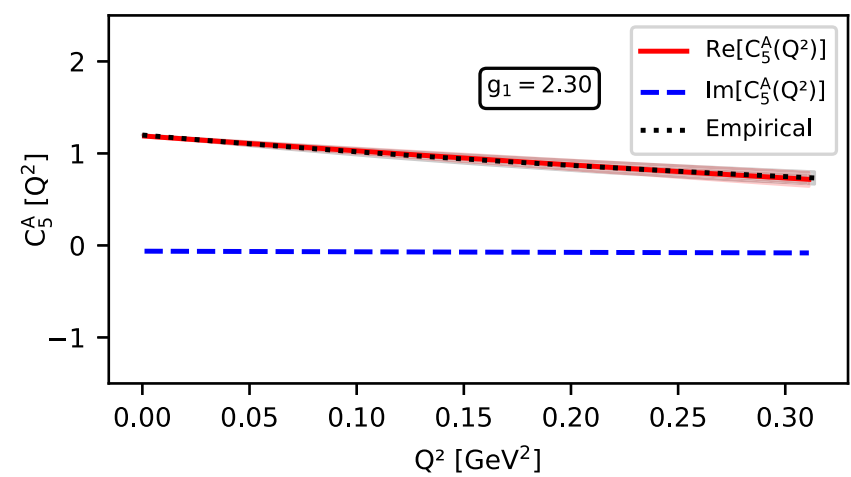

FIG. 4. Fit of the form factor $C_{5}^{A}\left(Q^{2}\right)$ for $g_{1}=2.30$. The red solid and blue dashed lines correspond to the real part and the imaginary part, respectively. The black dotted line is the empirical parametrization of the Adler model. The pink error band of the red solid line overlaps the narrower gray error band of dotted line.

which has to be compared with $\left\langle r_{A N \Delta}^{2}\right\rangle=0.427 \mathrm{fm}^{2}$ of the empirical parametrization. A smaller axial radius for the fit was to be expected, because the empirical parametrization contains more curvature while the fit behaves essentially linearly. Therefore, as can be seen from Fig. 4, the fit generates a slightly flatter behavior as a function of $Q^{2}$.

As no reliable experimental data exist for $\left\langle r_{A N \Delta}^{2}\right\rangle$, we also quote for comparison the empirical values for the mean-square axial radius for the nucleon: $\left\langle r_{A}^{2}\right\rangle=(0.444 \pm$ 0.018) $\mathrm{fm}^{2}$ [72] and $\left\langle r_{A}^{2}\right\rangle=(0.453 \pm 0.023) \mathrm{fm}^{2}$ [73]. In fact, based again on the quark-model relation of Eq. (32), the simplest assumption would be $C_{5}^{A}\left(Q^{2}\right)=\frac{3}{5} \sqrt{2} G_{A}\left(Q^{2}\right)$ [32], resulting in $\left\langle r_{A N \Delta}^{2}\right\rangle=\left\langle r_{A}^{2}\right\rangle$.

Instead of using the quark-model estimate for $g_{1}$, we also made use of the value $g_{1}=-1.21$ which was obtained in Ref. [74] from a fit to the $\pi N$ phase shifts of the $S$ and $P$ waves. As stated in Ref. [74], since $g_{1}$ appears only in the loop contribution of their calculation, a precise determination of its value is not to be expected. Note, in particular, that $g_{1}$ comes out with an opposite sign relative to the quark-model estimate. Using $g_{1}=-1.21$, we obtain for the LECs $\beta, \gamma$, and $\delta$ the values

$$
\begin{aligned}
& \beta=-0.0691, \quad \gamma=-19.8 \mathrm{GeV}^{-2}, \\
& \delta=-1.22 \mathrm{GeV}^{-2} .
\end{aligned}
$$

Here, the individual contributions to $C_{5}^{A}(0)$ are given by

$$
\begin{aligned}
C_{5}^{A}(0) & =1.19=\mathrm{g}+\gamma M_{\pi}^{2}+\text { loops } \\
& =1.08-0.36+0.47 .
\end{aligned}
$$

The individual contributions to $C_{5}^{A}\left(Q^{2}\right)$ for $g_{1}=-1.21$ are shown in Fig. 3(b). In the range $0 \leq Q^{2} \leq 0.3 \mathrm{GeV}^{2}$, the total result for $g_{1}=-1.21$ deviates from that for $g_{1}=2.30$ by less than $1 \%$. However, the loop contribution behaves

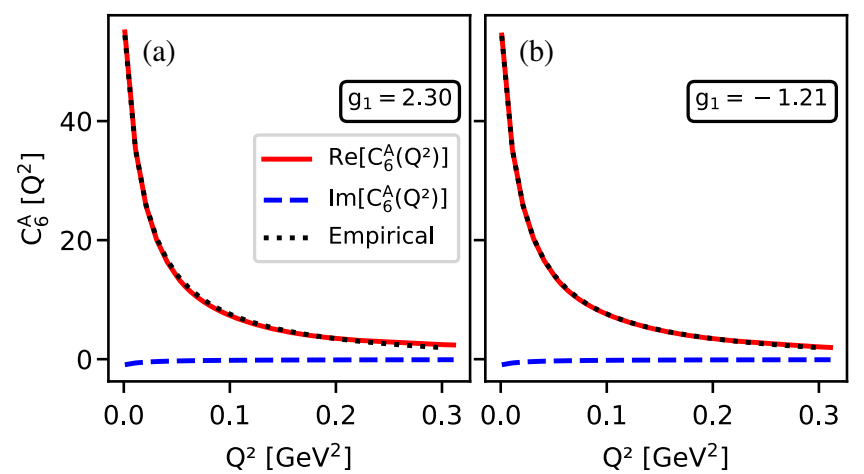

FIG. 5. Fit of the form factor $C_{6}^{A}\left(Q^{2}\right)$ for $g_{1}=2.30$ (left panel) and $g_{1}=-1.21$ (right panel), respectively. The red solid and blue dashed lines correspond to the real part and the imaginary part, respectively. The black dotted line is the pion-pole-dominated empirical parametrization with $C_{5}^{A}\left(Q^{2}\right)$ of the Adler model.

very differently, namely, at $Q^{2}=0$ it starts at a much lower value and it decreases with increasing $Q^{2}$ as opposed to a (stronger) increase with $Q^{2}$ for $g_{1}=2.30$. In the end, this behavior is compensated by the rather different values of $\gamma$ and $\delta$.

The results for $C_{6}^{A}\left(Q^{2}\right)$ were obtained by fitting to the pion-pole-dominated expression,

$$
C_{6}^{A}\left(Q^{2}\right)=m_{N}^{2} \frac{C_{5}^{A}\left(Q^{2}\right)}{M_{\pi}^{2}+Q^{2}}
$$

where $C_{5}^{A}\left(Q^{2}\right)$ is taken from Eq. (30). Such a fit contains two free parameters, namely, $\epsilon$ and $\zeta$, which were obtained as

$\epsilon=59.3 \mathrm{GeV}^{-2}$ and $\zeta=-11.5$ for $\mathrm{g}_{1}=2.30$,

$\epsilon=13.4 \mathrm{GeV}^{-2}$ and $\zeta=-3.40$ for $g_{1}=-1.21$.

The corresponding results for $C_{6}^{A}\left(Q^{2}\right)$ are shown in Fig. 5 . For $Q^{2} \lesssim 0.16 \mathrm{GeV}^{2}$, the fit for $\mathrm{g}_{1}=2.30$ is below the empirical form factor and deviates by less than $2.6 \%$ from the empirical $C_{6}^{A}\left(Q^{2}\right)$. Beyond $Q^{2} \approx 0.16 \mathrm{GeV}^{2}$, the fit is above the empirical result with a continuously increasing deviation reaching $-23 \%$ at $Q^{2}=0.3 \mathrm{GeV}^{2}$. For $g_{1}=-1.21$, the fit is generally closer to the empirical result than for $g_{1}=2.30$. For $Q^{2} \lesssim 0.06 \mathrm{GeV}^{2}$, the fit is below the empirical form factor, beyond $Q^{2}=0.06 \mathrm{GeV}^{2}$ it is above. Again, the maximal deviation happens at $Q^{2}=0.3 \mathrm{GeV}^{2}$ and amounts to $-3.2 \%$.

Given the results for the form factors $C_{5}^{A}\left(Q^{2}\right)$ and $C_{6}^{A}\left(Q^{2}\right)$, we are now also in the position to discuss the $\pi N \Delta$ transition form factor $G_{\pi N \Delta}\left(Q^{2}\right)$ of Eq. (12) (see Fig. 6). For $g_{1}=2.30$, our result deviates very quickly with increasing $Q^{2}$ from the pion-pole-dominated empirical result, 


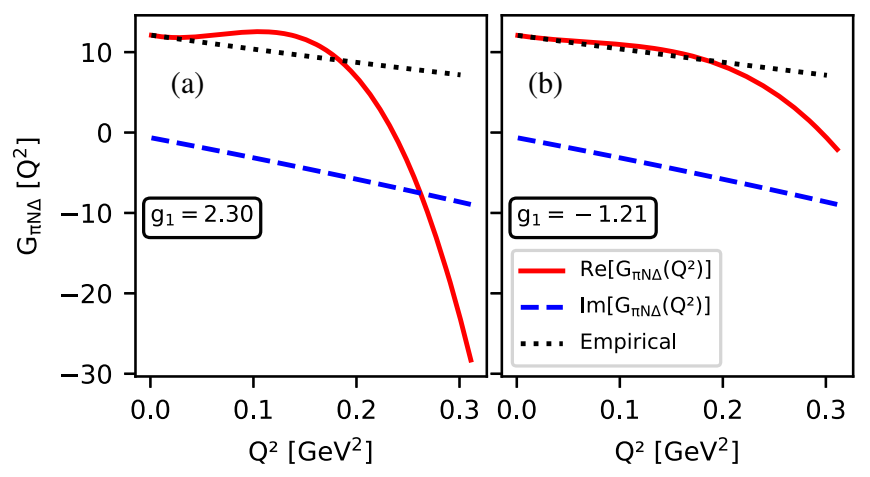

FIG. 6. Results for the transition form factor $G_{\pi N \Delta}\left(Q^{2}\right)$ of Eq. (12) for $g_{1}=2.30$ (left panel) and $g_{1}=-1.21$ (right panel), respectively. The red solid and blue dashed lines correspond to the real part and the imaginary part, respectively. The black dotted line is the empirical result using $C_{5}^{A}\left(Q^{2}\right)$ and $C_{6}^{A}\left(Q^{2}\right)$ of the Adler model.

$$
G_{\pi N \Delta}\left(Q^{2}\right)=\frac{m_{N}}{F_{\pi}} C_{5}^{A}\left(Q^{2}\right)
$$

In fact, the linear combination of Eq. (12) involves a delicate interplay between the terms

$$
\frac{m_{N}}{F_{\pi}} \frac{Q^{2}}{M_{\pi}^{2}} C_{5}^{A}\left(Q^{2}\right) \quad \text { and } \quad-\frac{m_{N}}{F_{\pi}} \frac{Q^{2}}{m_{N}^{2}} \frac{M_{\pi}^{2}+Q^{2}}{M_{\pi}^{2}} C_{6}^{A}\left(Q^{2}\right)
$$

The strong downward trend for increasing $Q^{2} \gtrsim 0.15 \mathrm{GeV}^{2}$ is due to a relatively large negative contribution proportional to $\left(Q^{2}\right)^{3}$, originating from the second term. The situation is somewhat better for $g_{1}=-1.21$, where the deviation starts at $Q^{2} \approx 0.2 \mathrm{GeV}^{2}$. This does not come as a surprise, because both $C_{5}^{A}\left(Q^{2}\right)$ and, in particular, $C_{6}^{A}\left(Q^{2}\right)$ are better described for $g_{1}=-1.21$. For the $\pi N \Delta$ coupling constant, we obtain

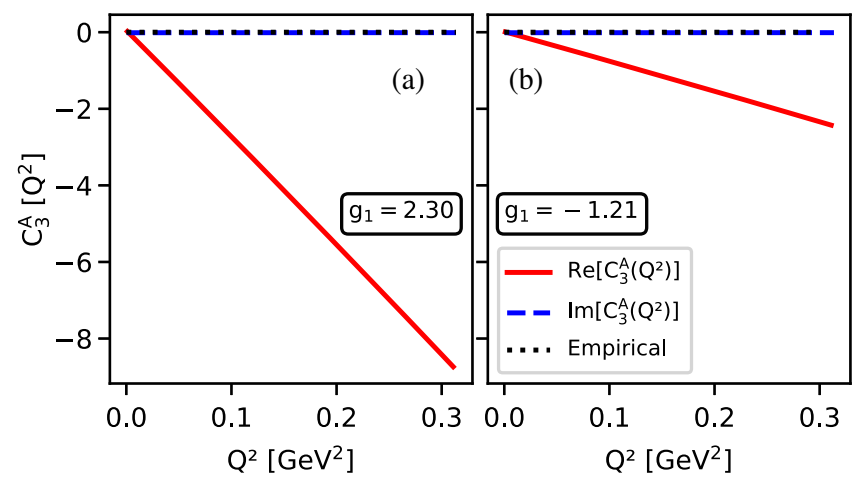

FIG. 7. Form factor $C_{3}^{A}\left(Q^{2}\right)$ for $g_{1}=2.30$ (left panel) and $g_{1}=$ -1.21 (right panel), respectively. The red solid and blue dashed lines correspond to the real part and the imaginary part, respectively. In the Adler model, the form factor is set to zero (black dotted lines).

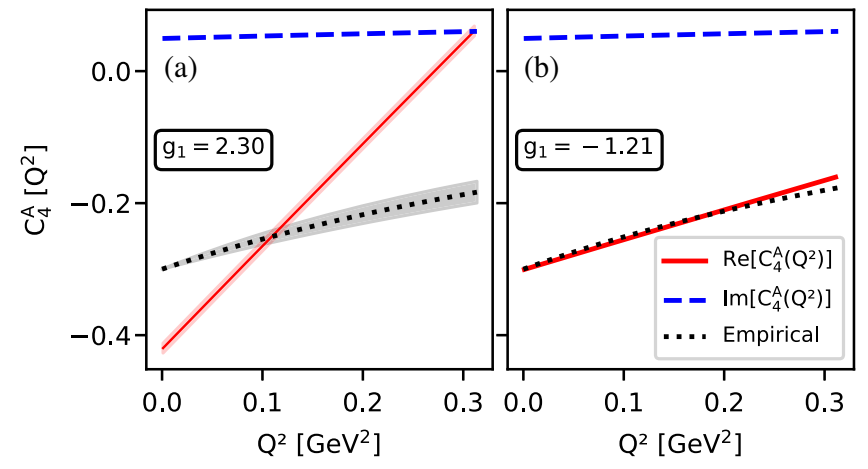

FIG. 8. Form factor $C_{4}^{A}\left(Q^{2}\right)$ for $g_{1}=2.30$ (left panel, including error bands) and $g_{1}=-1.21$ (right panel), respectively. The red solid and blue dashed lines correspond to the real part and the imaginary part, respectively. The black dotted line is the empirical parametrization of the Adler model.

$$
\begin{aligned}
G_{\pi N \Delta}\left(-M_{\pi}^{2}\right) & =g_{\pi N \Delta}=12.8 \quad \text { for } g_{1}=2.30 \\
g_{\pi N \Delta} & =12.5 \quad \text { for } g_{1}=-1.21
\end{aligned}
$$

These values result in a generalized Goldberger-Treiman discrepancy of

$$
\Delta=0.0533 \quad \text { and } \quad \Delta=0.0305 \text {, }
$$

for $g_{1}=2.30$ and $g_{1}=-1.21$, respectively. Even though the imaginary parts of $C_{5}^{A}\left(Q^{2}\right)$ and $C_{6}^{A}\left(Q^{2}\right)$ are small, we obtain a noticeable imaginary part for $G_{\pi N \Delta}\left(Q^{2}\right)$. In the present case, the imaginary part originates entirely from the loop contributions. In this context, one should keep in mind that, in the complex-mass scheme, the low-energy constants can also be complex numbers. Therefore, in principle, they could generate additional imaginary contributions. As our empirical ansatz for the form factors is real, we only fitted the real part of the form factors and left the imaginary tree-level contributions unspecified.

Finally, we turn to the form factors $C_{3}^{A}\left(Q^{2}\right)$ and $C_{4}^{A}\left(Q^{2}\right)$. These form factors have no analogue in the nucleon case. In Fig. 7, we show the loop contribution to $C_{3}^{A}\left(Q^{2}\right)$. The parameter $\alpha$ of Eq. (27) serves to shift the whole curve up or down and has been set to zero in the figure. No matter what the value for $\alpha$ is, our result is incompatible with the empirical ansatz $C_{3}^{A}\left(Q^{2}\right)=0$. By far the largest contribution to $C_{3}^{A}\left(Q^{2}\right)$ originates from diagram (t) of Fig. 2 and is proportional to $\mathrm{gg}_{1}^{2}$. Therefore, the case $\mathrm{g}_{1}=2.30$ produces a much stronger (negative) slope than $g_{1}=-1.21$. The result for $C_{4}^{A}\left(Q^{2}\right)$ is shown in Fig. 8. Here, we obtain a good description of the empirical form factor for $g_{1}=-1.21$, while the case $g_{1}=2.30$ again produces a much stronger (positive) slope. 


\section{COMPARISON WITH OTHER WORK}

Before we compare our calculation of the axial-vector current nucleon-to-delta transition form factors with other work, let us make a few general comments about the limitations of our results. Our calculation is a one-loop approximation at $\mathcal{O}\left(q^{3}\right)$. A complete one-loop calculation at $\mathcal{O}\left(q^{4}\right)$ would involve loop diagrams with a vertex from $\mathcal{L}_{\pi N \Delta}^{(2)}$ and, possibly, additional tree-level terms. As in the calculation of the nucleon axial-vector form factors, we expect from the loop diagrams nonanalytical corrections of $M_{\pi}^{3}$ to $C_{5}^{A}\left(Q^{2}\right)$ at $Q^{2}=0$ and also to $g_{\pi N \Delta}$ [see Eqs. (23), (24) and (37), (38) of Ref. [55] ], but no modification of the $Q^{2}$ behavior. Regarding the pion-mass dependence, we expect a reasonable convergence along the line of the twoloop analysis of $g_{A}$ in Ref. [75]. Since we cannot predict the values of the form factors at $Q^{2}=0$ anyway, the additional terms do not matter to our analysis. Of course, the knowledge of such terms would be of interest for extrapolations in the context of lattice QCD. In the case of the $Q^{2}$ behavior, the situation is more complex. Here, we expect that curvature terms might become important beyond approximately $Q^{2}=0.1 \mathrm{GeV}^{2}$ [55,76,77]. Such terms arise at the two-loop level in terms of loop contributions and new contact terms. However, from a phenomenological point of view, combining a one-loop calculation with meson exchange contributions such as in Ref. [55] might be a more efficient approach. For example, let us assume that the exchange of an $a_{1}$ axial-vector meson with mass $M_{a_{1}}=1.26 \mathrm{GeV}$ is the dominant mechanism contributing to $C_{5}^{A}\left(Q^{2}\right)$. From expanding the propagator $1 /\left(M_{a_{1}}^{2}+Q^{2}\right)$ as $M_{a_{1}}^{-2}\left(1-Q^{2} / M_{a_{1}}^{2}+\left(Q^{2} / M_{a_{1}}^{2}\right)^{2}+\cdots\right)$, we would expect a relative factor of the order of $Q^{2} / M_{a_{1}}^{2}$ multiplying $C_{5}^{A}\left(Q^{2}\right)-C_{5}^{A}(0)$. At $Q^{2}=0.3 \mathrm{GeV}^{2}$, this amounts to approximately $20 \%$, or about 0.09 in absolute terms for the magnitude of $C_{5}^{A}$.

Let us now turn to the comparison with other work. First, we compare our calculation with another calculation in the framework of covariant chiral perturbation theory [30]. Our starting point is different in that we are using the isovectorisospinor representation for the $\Delta$ involving the projection operator $\xi$, whereas Ref. [30] directly uses an isospin quadruplet. Furthermore, we have one more effective parameter than Ref. [30], which affects the tree-level result of $C_{6}^{A}\left(Q^{2}\right)$. The parameters entering the loop diagrams are essentially the same, in particular, Ref. [30] also uses the quark-model prediction $H_{A}=g_{1}=\frac{9}{5} g_{A}$. Nevertheless, our loop contributions are, in general, substantially larger in magnitude; this is particularly true for $C_{3}^{A}\left(Q^{2}\right)$. On the other hand, for both $g_{1}=2.30$ and $g_{1}=-1.21$, our total result for $C_{5}^{A}\left(Q^{2}\right)$ is closer to the empirical parametrization than the result of Ref. [30], in particular for $Q^{2} \gtrsim 0.1 \mathrm{GeV}^{2}$, whereas $C_{6}^{A}\left(Q^{2}\right)$ turns out to be very similar. For $C_{3}^{A}\left(Q^{2}\right)$ we obtain an opposite sign in comparison to Ref. [30].
Finally, for $C_{4}^{A}\left(Q^{2}\right)$, an ambiguous situation arises. For $g_{1}=-1.21$ there is a very good correspondence with the empirical form factor, whereas the result of Ref. [30] is substantially below the empirical form factor. However, for $g_{1}=2.30$, our result increases too quickly, yielding too large a slope.

In the framework of nonrelativistic chiral effective field theory to leading one-loop order [31], the $Q^{2}$ dependence of the form factor $C_{5}^{A}\left(Q^{2}\right)$ to order three is entirely generated by an LEC. The $Q^{2}$ dependence of the form factor $C_{6}^{A}\left(Q^{2}\right)$ is given by the pion-pole contribution and the same LEC as in $C_{5}^{A}\left(Q^{2}\right)$. Finally, $C_{3}^{A}\left(Q^{2}\right)$ and $C_{4}^{A}\left(Q^{2}\right)$ gain $Q^{2}$ and $M_{\pi}$ dependence only at higher orders. In particular, the prediction $C_{4}^{A}(0)=0$ (no LEC available in Ref. [31]) does not agree with our findings. However, based on the discussion in Appendix, the heavy-baryon Lagrangian $\mathcal{L}_{\pi N \Delta}^{(3)}$ at $\mathcal{O}\left(q^{3}\right)$ should also make a contribution to $C_{4}^{A}(0)$ [see Eq. (A4)]. Moreover, we find for all form factors a numerically significant loop contribution, in particular also from diagram (t) leading to the sensitivity on the sign of $g_{1}$. In the case of $C_{5}^{A}\left(Q^{2}\right)$ and $C_{6}^{A}\left(Q^{2}\right)$, the $Q^{2}$-dependent loop contributions are combined with contact terms, whereas for $C_{3}^{A}\left(Q^{2}\right)$ and $C_{4}^{A}\left(Q^{2}\right)$ the $Q^{2}$ dependence originates entirely from the loop diagrams. The different behavior of the loop contributions is not in conflict with the heavy-baryon calculation, where they should show up at a higher order in the combined chiral and inverse (large) mass expansion. Similar to the case of the determination of the nucleon electromagnetic form factors [76,77], a covariant calculation leads to the fact that higherorder terms are retained that do not (yet) occur in the inverse mass expansion. Our values for the generalized Goldberger-Treiman discrepancy are slightly larger than the $\sim 2 \%$ predicted in the framework of heavy-baryon chiral perturbation theory [29].

The inclusion of the $a_{1}$ meson as an explicit dynamical degree of freedom was discussed in Ref. [32]. Besides the $a_{1}$-meson mass, this introduces one additional effective parameter. The $a_{1}$ meson effects the shape of the form factors $C_{5}^{A}\left(Q^{2}\right)$ and $C_{6}^{A}\left(Q^{2}\right)$; the form factor $C_{5}^{A}\left(Q^{2}\right)$ develops more curvature and $C_{6}^{A}\left(Q^{2}\right)$ lies above the pion-pole dominance prediction for $Q^{2} \gtrsim 0.1 \mathrm{GeV}^{2}$.

Results from lattice QCD for $C_{5}^{A}\left(Q^{2}\right)$ and $C_{6}^{A}\left(Q^{2}\right)$ were reported in Refs. [34,35]. In general, the values of $C_{5}^{A}(0)$ in their calculations come out as smaller than one, i.e., they are also smaller than our results. At the same time, the axial mass turns out to be larger than $M_{A}=1.28 \mathrm{GeV}$, corresponding to a smaller mean-square axial transition radius. This corresponds to our findings. At low $Q^{2}$, the lattice results for $C_{6}^{A}\left(Q^{2}\right)$ turn out to be smaller than our results. Finally, Ref. [35] contains two fits to $G_{\pi N \Delta}\left(Q^{2}\right)$. For the coupling constant $g_{\pi N \Delta}$, which Ref. [35] defines at $Q^{2}=0$ rather than $Q^{2}=-M_{\pi}^{2}$, values between 8.44 and 16.3 are 
obtained, which have to be compared with our values 12.8 and 12.5 .

As a representative example for studies in the framework of nonrelativistic (chiral) quark models, we take the results of Ref. [28]. First, we observe that the predictions of the form factors in the quark model are real quantities instead of complex functions in our calculation. The dominant form factor $C_{5}^{A}\left(Q^{2}\right)$ starts with $C_{5}^{A}(0)=0.93$ and produces curvature according to an axial-vector meson dominance coupling (at the quark level) similar to Ref. [32]. The axial radius is predicted as $\left\langle r_{A N \Delta}^{2}\right\rangle=0.59 \mathrm{fm}^{2}$. They also predict a nonzero form factor $C_{3}^{A}\left(Q^{2}\right)$ in the low- $Q^{2}$ region, but of significantly smaller magnitude.

Besides Ref. [21], from which we took the empirical parametrization of the form factors, some more recent experimental information is available for the linear combination $\quad G_{N \Delta}^{A}\left(Q^{2}\right)=\frac{1}{2}\left[m_{N}^{2}-m_{\Delta}^{2}+Q^{2}\right] C_{4}^{A}\left(Q^{2}\right)-$ $m_{N}^{2} C_{5}^{A}\left(Q^{2}\right)$, which was extracted from the parityviolating asymmetry in inelastic electron-nucleon scattering near the $\Delta$ resonance. Reference [22] reports ${ }^{14}$ $G_{N \Delta}^{A}\left(Q^{2}=0.34 \mathrm{GeV}^{2}\right)=-0.05 \pm(0.35)_{\text {stat }} \pm(0.34)_{\text {sys }} \pm$ $(0.06)_{\text {theory }}$, whereas we obtained $-0.584 \mathrm{GeV}^{2}$ for $\mathrm{g}_{1}=$ 2.30 and $-0.544 \mathrm{GeV}^{2}$ for $\mathrm{g}_{1}=-1.21$.

\section{SUMMARY}

We analyzed the low- $Q^{2}$ behavior of the axial-vector nucleon-to-delta transition form factors $C_{3}^{A}\left(Q^{2}\right), C_{4}^{A}\left(Q^{2}\right)$, $C_{5}^{A}\left(Q^{2}\right)$, and $C_{6}^{A}\left(Q^{2}\right)$ at the one-loop level of relativistic baryon chiral perturbation theory. In total, the calculation involves six free parameters $\alpha, \beta, \gamma, \delta, \epsilon$, and $\zeta$ [see Eqs. (27)]. The constants $g_{A}, F_{\pi}, M_{\pi}, m_{N}$, and $z_{\Delta}$ were fixed in terms of their empirical values. For the coupling constant $g$ we made use of the quark-model prediction $\mathrm{g}=\frac{3}{5} \sqrt{2} g_{A}$. Finally, for the coupling constant $g_{1}$, appearing in certain loop diagrams only, we considered two scenarios: we either made use of the quark-model prediction $g_{1}=\frac{9}{5} g_{A}=2.30$ or of $g_{1}=-1.21$ as obtained from an analysis of the $\pi N$ phase shifts of the $S$ and $P$ waves. Since there is essentially no direct experimental information on the form factors available, we took the empirical parametrizations used in the analysis of Ref. [21] to determine our parameters. For our fits, we chose the interval $0 \leq Q^{2} \leq 0.3136 \mathrm{GeV}^{2}$, where the upper end of the interval is likely to be at the verge of the applicability of a one-loop calculation. For the form factor $C_{5}^{A}\left(Q^{2}\right)$ we obtain good descriptions for both $g_{1}=2.30$ and $g_{1}=-1.21$, deviating from the empirical form by less than $3 \%$ and $1 \%$, respectively. As can be seen from Fig. 3, the loop corrections are sizable and their slope depends on

\footnotetext{
${ }^{14}$ Reference [22] does not quote any units, even though in natural units the linear combination has dimension energy squared.
}

the sign of $g_{1}$. As a consequence, the total result involves a delicate interplay between the loop contributions and the parameters $\gamma$ and $\delta$ [see Eqs. (33) and (36)]. The parameters $\epsilon$ and $\zeta$ were determined from the fit to $C_{6}^{A}\left(Q^{2}\right)$, where, again, for $g_{1}=-1.21$ our result is closer to the empirical form factor than for $g_{1}=2.30$ (see Fig. 5). As a result, the $\pi N \Delta$ transition form factor $G_{\pi N \Delta}\left(Q^{2}\right)$ deviates from the simple expectation $G_{\pi N \Delta}\left(Q^{2}\right)=\frac{m_{N}}{F_{\pi}} C_{5}^{A}\left(Q^{2}\right)$, again, more so for $g_{1}=2.30$ than for $g_{1}=-1.21$ (see Fig. 6). For the $\pi N \Delta$ coupling constant we obtained $g_{\pi N \Delta}=12.8$ for $g_{1}=$ 2.30 and $g_{\pi N \Delta}=12.5$ for $g_{1}=-1.21$, resulting in the Goldberger-Treiman discrepancies $\Delta=0.0533$ and $\Delta=0.0305$, respectively. The parameters $\alpha$ and $\beta$ are responsible for vertically shifting the curves of the form factors $C_{3}^{A}\left(Q^{2}\right)$ and $C_{4}^{A}\left(Q^{2}\right)$, respectively, they cannot, however, modify their shapes. Therefore, the loop contributions are, to some extent, a unique feature of the predictions for $C_{3}^{A}\left(Q^{2}\right)$ and $C_{4}^{A}\left(Q^{2}\right)$. In particular, our calculation predicts $C_{3}^{A}\left(Q^{2}\right)$ to be different from zero in contrast to the empirical parametrization $C_{3}^{A}\left(Q^{2}\right)=0$ (see Fig. 7). Moreover, for $g_{2}=-1.21$ we obtain a very good agreement between our result for $C_{3}^{A}\left(Q^{2}\right)$ and the empirical form factor (see Fig. 8). A somewhat surprising feature is the fact that the negative value of $g_{1}$ in all cases gives a better agreement with the empirical form factors than the quark-model result which uniquely predicts a positive sign. Unfortunately, as in the case of $\pi N$ scattering, $g_{1}$ does not enter the calculation at leading order but only at the loop level. More about the sign of $g_{1}$ could possibly be learned from radiative pion-nucleon scattering $\pi N \rightarrow \pi \gamma N$ or radiative pion photoproduction $\gamma N \rightarrow \gamma \pi N$ in the $\Delta$-resonance region, where the $\pi \Delta \Delta$ vertex contributes at tree level and thus at leading order. Finally, our results at the one-loop level suggest that the imaginary parts of the axial-vector nucleon-to-delta transition form factors are, in general, rather small. This is a striking difference to the electromagnetic transition form factors, where only in the case of the magnetic dipole form factor $G_{M}\left(Q^{2}\right)$ the imaginary part is negligibly small compared to the real part, whereas for the electric dipole and charge quadrupole form factors $G_{E}\left(Q^{2}\right)$ and $G_{C}\left(Q^{2}\right)$ the real and imaginary parts are of the same order of magnitude [23].

\section{ACKNOWLEDGMENTS}

The authors would like to thank J. Gegelia for valuable discussions.

\section{APPENDIX: $\mathcal{O}\left(\boldsymbol{q}^{2}\right)$ LAGRANGIAN}

For our calculation, we need the pion-nucleon-delta interaction vertex and the axial-vector-nucleon-delta interaction vertex. The building blocks that potentially contribute are the chiral vielbein [see Eqs. (22) and (23)] and 


$$
\begin{aligned}
\chi_{-} & =u^{\dagger} \chi u^{\dagger}-u \chi^{\dagger} u \rightarrow M^{2}\left(U^{\dagger}-U\right) \rightarrow-2 i M^{2} \frac{\tau_{i} \phi_{i}}{F}, \quad \text { (A1) } \\
f_{-\mu \nu} & =u f_{L \mu \nu} u^{\dagger}-u^{\dagger} f_{R \mu \nu} u \rightarrow f_{L \mu \nu}-f_{R \mu \nu} \rightarrow-2\left(\partial_{\mu} a_{\nu}-\partial_{\nu} a_{\mu}\right) \\
& =-\tau_{i}\left(\partial_{\mu} a_{\nu, i}-\partial_{\nu} a_{\mu, i}\right) .
\end{aligned}
$$

One also has to consider covariant derivatives of these building blocks.

According to Jiang et al. [64], the Lagrangian at $\mathcal{O}\left(q^{2}\right)$, $\mathcal{L}_{\pi N \Delta}^{(2)}$, contains three structures [see Eq. (66) of Ref. [64] ]. The first two structures are proportional to the product $u_{\mu, i} u_{\nu, j}$ and thus contribute neither to the $\pi N \Delta$ interaction vertex nor to the $a N \Delta$ interaction vertex. The third structure is proportional to $f_{+\mu \nu}$ which contributes to the $v N \Delta$ interaction vertex but not to the $a N \Delta$ interaction vertex. In other words, according to Jiang et al., there are no contact interaction contributions to the transition form factors at $\mathcal{O}\left(q^{2}\right)$.

Jiang et al. compare their results with Ref. [60], which they quote as their Eq. (67). However, Hemmert et al. [60] did not construct the covariant version but rather the heavybaryon version of the Lagrangian. According to Eq. (82) of Ref. [60], they factor out $\exp \left(-i M_{0} v \cdot x\right)$ with a common mass $M_{0}$ for both the nucleon field and the $\Delta$ field. The relevant heavy-baryon Lagrangian is then given in Eq. (112), where $N$ and $T$ are heavy-baryon fields. Note that there is no covariant Lagrangian in Ref. [60]. In other words, Jiang et al. must have reconstructed their Eq. (67) from the heavy-baryon Lagrangian. We make use of the results of section 5.5. of Ref. [56] to establish the connection. Using Eq. (5.122) of Ref. [56], a single term $v^{\mu}$ originates from $\gamma^{\mu}$ and $2 S^{\mu}$ from $\gamma^{\mu} \gamma_{5}$, respectively.

Let us have a look at the first term of Eq. (112) of Hemmert et al. This should result from

$$
\begin{aligned}
& \frac{1}{2 M_{0}} \bar{\Psi}_{i}^{\mu} b_{1} i f_{+\mu \nu, i} \frac{1}{2} \gamma^{\nu} \gamma_{5} \Psi+\text { H.c. } \\
& =\frac{1}{2 M_{0}}\left(-\frac{1}{2} b_{1} i \bar{\Psi}_{i}^{\mu} f_{+\mu \nu, i} \frac{1}{2} \gamma_{5} \gamma^{\nu} \Psi+\text { H.c. }\right),
\end{aligned}
$$

which, apart from a factor $1 /\left(2 M_{0}\right)$, agrees with the first term of Eq. (67) of Jiang et al. ${ }^{15}$ Now let us turn to the second term of Hemmert et al. which should originate from

$$
\frac{1}{2 M_{0}} \bar{\Psi}_{i}^{\mu} i b_{2} f_{-\mu \nu, i} \gamma^{\nu} \Psi+\text { H.c. }
$$

Comparing with Eq. (67) of Jiang et al., we see that they took the wrong operator $D^{\nu}$ instead of $\gamma^{\nu}$ and then argue that such a term can be eliminated using arguments given in

\footnotetext{
${ }^{15} \mathrm{We}$ left out the projector $O_{A, n}^{\mu \nu}\left(z_{n}\right)=g^{\mu \nu}+\left(z_{n}+\frac{1}{2}(1+\right.$ $\left.\left.4 z_{n}\right) A\right) \gamma^{\mu} \gamma^{\nu}$ as well as the projector $\xi_{i j}^{\frac{3}{2}}$.
}

a previous section of Ref. [64]. In fact, Holmberg and Leupold [65] also obtain a structure analogous to the $b_{2}$ term in their construction for the decuplet-to-octet transition Lagrangian at next-to-leading order.

We will now show that the $b_{2}$ term gives an explicit contribution to the form factor $C_{3}^{A}\left(Q^{2}\right)$ at $\mathcal{O}\left(q^{2}\right)$. Using Eq. (A2) and after dropping the factor $1 /\left(2 M_{0}\right)$, from the $b_{2}$ term we obtain the Lagrangian

$$
i b_{2} \bar{\Psi}_{\lambda, i} \xi_{i j}^{\frac{3}{2}}\left(-\partial^{\lambda} a_{j}^{\mu}+\partial^{\mu} a_{j}^{\lambda}\right) \gamma_{\mu} \Psi+\text { H.c. },
$$

resulting in the form factor contribution

$$
\begin{gathered}
i b_{2} \bar{w}_{\lambda}\left(\left(-\left(-i q^{\lambda}\right)\right) \epsilon^{\mu}+\left(-i q^{\mu}\right) \epsilon^{\lambda}\right) \gamma_{\mu} u \\
=i b_{2} \bar{w}_{\lambda}\left(i q^{\lambda} \gamma^{\mu}-i \not g g^{\lambda \mu}\right) u \epsilon_{\mu} \\
=-b_{2} \bar{w}_{\lambda}\left(q^{\lambda} \gamma^{\mu}-\not q g^{\lambda \mu}\right) u \epsilon_{\mu} \\
=m_{N} b_{2} \bar{w}_{\lambda} \frac{\not g^{\lambda \mu}-q^{\lambda} \gamma^{\mu}}{m_{N}} u \epsilon_{\mu},
\end{gathered}
$$

from which we obtain the contribution $m_{N} b_{2} \equiv \alpha$ to the form factor $C_{3}^{A}\left(Q^{2}\right)$.

Furthermore, we can relate the contribution to the form factor $C_{4}^{A}\left(Q^{2}\right)$ to the Lagrangian

$$
\frac{\beta}{m_{N}^{2}} D_{\mu} \bar{\Psi}_{\lambda, i} \xi_{i j}^{\frac{3}{2}} f_{-j}^{\lambda \mu} \Psi+\text { H.c. },
$$

resulting in the invariant matrix element

$$
\mathcal{M}=i \frac{\beta}{m_{N}^{2}} \epsilon_{\mu} \bar{w}_{\lambda}\left(g^{\lambda \mu} p_{f} \cdot q-q^{\lambda} p_{f}^{\mu}\right) u
$$

and, thus, the constant contribution $\beta$ to $C_{4}^{A}\left(Q^{2}\right)$. In fact, Holmberg and Leupold [65] showed how to make use of a total-derivative argument and the lowest-order equation of motion such that the Lagrangian of Eq. (A3) can be reexpressed in terms of the $b_{2}$ Lagrangian and terms of the $\mathcal{O}\left(q^{3}\right)$ Lagrangian. For the purpose of simplicity, we omit the projector $\xi$, the Cartesian isospin indices and the term H.c., because they are not relevant for the argument. The reduction proceeds as follows:

$$
D_{\mu} \bar{\Psi}_{\lambda} f_{-}^{\lambda \mu} \Psi=\partial_{\mu}\left(\bar{\Psi}_{\lambda} f_{-}^{\lambda \mu} \Psi\right)-\bar{\Psi}_{\lambda} D_{\mu} f_{-}^{\lambda \mu} \Psi-\bar{\Psi}_{\lambda} f_{-}^{\lambda \mu} D_{\mu} \Psi .
$$

The first term is a total derivative and, thus, does not contribute to the dynamics, the second term contributes to the $\delta$ term proportional to $Q^{2}$ in Eq. (27). Therefore, we continue with only the third term, 


$$
\begin{aligned}
-\bar{\Psi}_{\lambda} f_{-}^{\lambda \mu} D_{\mu} \Psi & =-\bar{\Psi}_{\lambda} f_{-}^{\lambda \mu} g_{\mu \nu} D^{\nu} \Psi \\
& =-\frac{1}{2} \bar{\Psi}_{\lambda} f_{-}^{\lambda \mu}\left(\gamma_{\mu} \gamma_{\nu}+\gamma_{\nu} \gamma_{\mu}\right) D^{\nu} \Psi \\
& =-\frac{1}{2} \bar{\Psi}_{\lambda} f_{-}^{\lambda \mu} \gamma_{\mu} \not \Phi \Psi-\frac{1}{2} \bar{\Psi}_{\lambda} f_{-}^{\lambda \mu} \gamma_{\nu} \gamma_{\mu} D^{\nu} \Psi
\end{aligned}
$$

Using the lowest-order equation of motion (or an appropriate field redefinition, see Ref. [78]), the first term is proportional to the $b_{2}$ term plus higher-order terms, and we continue with the second term:

$$
\begin{aligned}
-\frac{1}{2} \bar{\Psi}_{\lambda} f_{-}^{\lambda \mu} \gamma_{\nu} \gamma_{\mu} D^{\nu} \Psi= & -\frac{1}{2} \partial^{\nu}\left(\bar{\Psi}_{\lambda} f_{-}^{\lambda \mu} \gamma_{\nu} \gamma_{\mu} \Psi\right) \\
& +\frac{1}{2} D^{\nu} \bar{\Psi}_{\lambda} f_{-}^{\lambda \mu} \gamma_{\nu} \gamma_{\mu} \Psi+\frac{1}{2} \bar{\Psi}_{\lambda} D^{\nu} f_{-}^{\lambda \mu} \gamma_{\nu} \gamma_{\mu} \Psi .
\end{aligned}
$$

The first term is again a total derivative and the second term can be reduced to a $b_{2}$ term, using the delta equation of motion. Finally, we reexpress the third term as

$$
\frac{1}{2} \bar{\Psi}_{\lambda} D^{\nu} f_{-}^{\lambda \mu} \gamma_{\nu} \gamma_{\mu} \Psi=\frac{1}{2} \bar{\Psi}_{\lambda} D_{\mu} f_{-}^{\lambda \mu} \Psi-\frac{i}{2} \bar{\Psi}_{\lambda} D^{\nu} f_{-}^{\lambda \mu} \sigma_{\nu \mu} \Psi
$$

The first term is again a $\delta$ term and the second term is, last but not least, the independent structure without hard derivatives. Using, again, the results of Ref. [56], the heavy-baryon reduction of this term would look like

$$
-i \epsilon_{\mu \nu \rho \sigma} v^{\rho} \bar{T}_{\lambda} D^{\mu} f_{-}^{\lambda \nu} S^{\sigma} N
$$

As a matter of convenience, we will stick to the Lagrangian of Eq. (A3), because, for our purposes, it is only relevant to know that we have a free parameter at our disposal, even if it originates from the $\mathcal{O}\left(q^{3}\right)$ and which, only after rewriting, contributes to the transition matrix element in terms of $C_{4}^{A}\left(Q^{2}\right)$. Finally, it was shown in Ref. [65] that at $\mathcal{O}\left(q^{2}\right)$ there is no "new" contribution to the $\pi N \Delta$ vertex.
[1] P. A. Zyla et al. (Particle Data Group Collaboration), Prog. Theor. Exp. Phys. (2020), $083 \mathrm{C} 01$.

[2] W. Bartel, B. Dudelzak, H. Krehbiel, J. McElroy, U. Meyer-Berkhout, W. Schmidt, V. Walther, and G. Weber, Phys. Lett. 28B, 148 (1968).

[3] K. Baetzner et al., Phys. Lett. 39B, 575 (1972).

[4] S. Stein, W. B. Atwood, E. D. Bloom, R. L. A. Cottrell, H. DeStaebler, C. L. Jordan, H. G. Piel, C. Y. Prescott, R. Siemann, and R. E. Taylor, Phys. Rev. D 12, 1884 (1975).

[5] R. Beck et al., Phys. Rev. C 61, 035204 (2000).

[6] T. Pospischil et al., Phys. Rev. Lett. 86, 2959 (2001).

[7] C. Mertz et al., Phys. Rev. Lett. 86, 2963 (2001).

[8] K. Joo et al. (CLAS Collaboration), Phys. Rev. Lett. 88, 122001 (2002).

[9] N. F. Sparveris et al. (OOPS Collaboration), Phys. Rev. Lett. 94, 022003 (2005).

[10] D. Elsner et al., Eur. Phys. J. A 27, 91 (2006).

[11] J. J. Kelly et al., Phys. Rev. C 75, 025201 (2007).

[12] S. Stave et al. (A1 Collaboration), Phys. Rev. C 78, 025209 (2008).

[13] I. G. Aznauryan et al. (CLAS Collaboration), Phys. Rev. C 80, 055203 (2009).

[14] A. Blomberg et al., Phys. Lett. B 760, 267 (2016).

[15] L. Tiator, M. Döring, R. L. Workman, M. Hadžimehmedović, H. Osmanović, R. Omerović, J. Stahov, and A. Švarc, Phys. Rev. C 94, 065204 (2016).

[16] L. Tiator, D. Drechsel, S. S. Kamalov, and M. Vanderhaeghen, Eur. Phys. J. Special Topics 198, 141 (2011).

[17] I. G. Aznauryan and V. D. Burkert, Prog. Part. Nucl. Phys. 67, 1 (2012).

[18] S. J. Barish et al., Phys. Rev. D 19, 2521 (1979).

[19] G. M. Radecky et al., Phys. Rev. D 25, 1161 (1982); 26, 3297(E) (1982).
[20] T. Kitagaki et al., Phys. Rev. D 34, 2554 (1986).

[21] T. Kitagaki et al., Phys. Rev. D 42, 1331 (1990).

[22] D. Androic et al. (G0 Collaboration), arXiv:1212.1637.

[23] M. Hilt, T. Bauer, S. Scherer, and L. Tiator, Phys. Rev. C 97, 035205 (2018).

[24] J. G. Körner, T. Kobayashi, and C. Avilez, Phys. Rev. D 18, 3178 (1978).

[25] T. R. Hemmert, B. R. Holstein, and N. C. Mukhopadhyay, Phys. Rev. D 51, 158 (1995).

[26] J. Líu, N. C. Mukhopadhyay, and L. Zhang, Phys. Rev. C 52, 1630 (1995).

[27] B. Golli, S. Širca, L. Amoreira, and M. Fiolhais, Phys. Lett. B 553, 51 (2003).

[28] D. Barquilla-Cano, A. J. Buchmann, and E. Hernandez, Phys. Rev. C 75, 065203 (2007); 77, 019903(E) (2008).

[29] S. L. Zhu and M. J. Ramsey-Musolf, Phys. Rev. D 66, 076008 (2002).

[30] L. S. Geng, J. Martin Camalich, L. Alvarez-Ruso, and M. J. Vicente Vacas, Phys. Rev. D 78, 014011 (2008).

[31] M. Procura, Phys. Rev. D 78, 094021 (2008).

[32] Y. Ünal, A. Küçükarslan, and S. Scherer, Phys. Rev. D 99, 014012 (2019).

[33] C. Alexandrou, T. Leontiou, J. W. Negele, and A. Tsapalis, Phys. Rev. Lett. 98, 052003 (2007).

[34] C. Alexandrou, G. Koutsou, T. Leontiou, J. W. Negele, and A. Tsapalis, Phys. Rev. D 76, 094511 (2007); 80, 099901(E) (2009).

[35] C. Alexandrou, G. Koutsou, J. W. Negele, Y. Proestos, and A. Tsapalis, Phys. Rev. D 83, 014501 (2011).

[36] T. M. Aliev, K. Azizi, and A. Ozpineci, Nucl. Phys. A799, 105 (2008).

[37] A. Küçükarslan, U. Ozdem, and A. Ozpineci, Nucl. Phys. B913, 132 (2016). 
[38] J. Gegelia and S. Scherer, Eur. Phys. J. A 44, 425 (2010).

[39] C. Hacker, N. Wies, J. Gegelia, and S. Scherer, Phys. Rev. C 72, 055203 (2005).

[40] N. Wies, J. Gegelia, and S. Scherer, Phys. Rev. D 73, 094012 (2006).

[41] D. Djukanovic, J. Gegelia, A. Keller, and S. Scherer, Phys. Lett. B 680, 235 (2009).

[42] R. G. Stuart, Pitfalls of radiative corrections near a resonance, in $Z^{0}$ Physics, edited by J. Tran Thanh Van (Editions Frontières, Gif-sur-Yvette, 1990), p. 41.

[43] A. Denner, S. Dittmaier, M. Roth, and D. Wackeroth, Nucl. Phys. B560, 33 (1999).

[44] A. Denner and S. Dittmaier, Nucl. Phys. B, Proc. Suppl. 160, 22 (2006).

[45] S. Actis and G. Passarino, Nucl. Phys. B777, 100 (2007).

[46] S. Actis, G. Passarino, C. Sturm, and S. Uccirati, Phys. Lett. B 669, 62 (2008).

[47] J. D. Bjorken and S. D. Drell, Relativistic Quantum Fields (McGraw-Hill, New York, 1965).

[48] S. L. Adler, Ann. Phys. (N.Y.) 50, 189 (1968).

[49] J. Gasser and H. Leutwyler, Ann. Phys. (N.Y.) 158, 142 (1984).

[50] A. R. Edmonds, Angular Momentum in Quantum Mechanics, 2nd ed. (Princeton University Press, Princeton, N. J., 1960).

[51] W. Rarita and J. Schwinger, Phys. Rev. 60, 61 (1941).

[52] S. Kusaka, Phys. Rev. 60, 61 (1941).

[53] A. Agadjanov, V. Bernard, U.-G. Meißner, and A. Rusetsky, Nucl. Phys. B886, 1199 (2014).

[54] C. H. Llewellyn Smith, Phys. Rep. 3, 261 (1972).

[55] M. R. Schindler, T. Fuchs, J. Gegelia, and S. Scherer, Phys. Rev. C 75, 025202 (2007).

[56] S. Scherer, Adv. Nucl. Phys. 27, 277 (2003).

[57] S. L. Adler and R.F. Dashen, Current Algebras and Applications to Particle Physics (Benjamin, New York, 1968).

[58] J. Gasser, M. E. Sainio, and A. Švarc, Nucl. Phys. B307, 779 (1988).
[59] S. Scherer and M. R. Schindler, Lect. Notes Phys. 830, 1 (2012).

[60] T. R. Hemmert, B. R. Holstein, and J. Kambor, J. Phys. G 24, 1831 (1998).

[61] G. Colangelo, J. Gasser, and H. Leutwyler, Phys. Rev. Lett. 86, 5008 (2001).

[62] P. A. Moldauer and K. M. Case, Phys. Rev. 102, 279 (1956).

[63] L. M. Nath, B. Etemadi, and J. D. Kimel, Phys. Rev. D 3, 2153 (1971).

[64] S. Z. Jiang, Y. R. Liu, and H. Q. Wang, Phys. Rev. D 97, 014002 (2018).

[65] M. Holmberg and S. Leupold, Eur. Phys. J. A 54, 103 (2018).

[66] V. Pascalutsa and D. R. Phillips, Phys. Rev. C 67, 055202 (2003).

[67] M. L. Goldberger and S. B. Treiman, Phys. Rev. 110, 1178 (1958).

[68] Y. Nambu, Phys. Rev. Lett. 4, 380 (1960).

[69] H. Pagels, Phys. Rev. 179, 1337 (1969).

[70] P. A. Schreiner and F. Von Hippel, Nucl. Phys. B58, 333 (1973).

[71] T. Bauer, Y. Ünal, A. Küçükarslan, and S. Scherer, Phys. Rev. C 96, 025203 (2017).

[72] V. Bernard, L. Elouadrhiri, and U.-G. Meißner, J. Phys. G 28, R1 (2002).

[73] A. Bodek, S. Avvakumov, R. Bradford, and H. S. Budd, Eur. Phys. J. C 53, 349 (2008).

[74] D. L. Yao, D. Siemens, V. Bernard, E. Epelbaum, A. M. Gasparyan, J. Gegelia, H. Krebs, and U.-G. Meißner, J. High Energy Phys. 05 (2016) 038.

[75] V. Bernard and U.-G. Meißner, Phys. Lett. B 639, 278 (2006).

[76] B. Kubis and U.-G. Meißner, Nucl. Phys. A679, 698 (2001).

[77] T. Fuchs, J. Gegelia, and S. Scherer, J. Phys. G 30, 1407 (2004).

[78] S. Scherer and H. W. Fearing, Phys. Rev. D 52, 6445 (1995). 